\title{
Risk Stratification of Metastatic Neck Nodes by CT and PET in Patients with Head and Neck Cancer Receiving Definitive Radiotherapy
}

\author{
Ying-Chun Lin*1, Shang-Wen Chen*1-3 ${ }^{*}$ Te-Chun Hsieh ${ }^{4,5}$, Kuo-Yang Yen ${ }^{4,5}$, Shih-Neng Yang ${ }^{1,5}$, Yao-Ching Wang ${ }^{1}$, \\ and Chia-Hung $\mathrm{Kao}^{2,4}$ \\ ${ }^{1}$ Department of Radiation Oncology, China Medical University Hospital, Taichung, Taiwan; ${ }^{2}$ School of Medicine, China Medical \\ University, Taichung, Taiwan; ${ }^{3}$ School of Medicine, Taipei Medical University, Taipei, Taiwan; ${ }^{4}$ Department of Nuclear Medicine and \\ PET Center, China Medical University Hospital, Taichung, Taiwan; and ${ }^{5}$ Department of Biomedical Imaging and Radiological \\ Science, China Medical University, Taichung, Taiwan
}

\begin{abstract}
The aim of this study was to investigate the prognostic impact of CT and ${ }^{18} \mathrm{~F}-\mathrm{FDG}$ PET/CT on the outcome of metastatic neck node (MNN) in patients with head and neck cancer receiving definitive radiotherapy or chemoradiotherapy. Methods: This patient-based study included 91 patients diagnosed with pharyngeal cancers with MNN (N1, 15; N2, 70; N3, 6). All had pretreatment CT and PET/CT before definitive chemoradiotherapy/radiotherapy. Parameters of MNNs for each patient, including maximal diameter, nodal volume, radiologic central necrosis, maximum standardized uptake value, metabolic tumor volume, and total lesion glycolysis (TLG), were retrieved for the analysis. Nodal relapse-free survival (NRFS) and survivals were calculated using the Kaplan-Meier method. Independent predictors were identified using Cox regression analysis. Results: After a median follow-up of $18 \mathrm{mo}$, 64 patients remained nodal relapse-free, and 27 experienced neck recurrence. Multivariate analysis showed that the application of $40 \%$ of the maximal uptake of nodal TLG (N-TLG40\%) $38 \mathrm{~g}$ or greater $(P=0.03$; hazard ratio, 2.63; 95\% confidence interval, 1.10-6.30) and radiologic necrosis on CT scan ( $P=0.001$; hazard ratio, 10.99; 95\% confidence interval, 2.56-47.62) were 2 adverse features for NRFS. Patients who had an N-TLG40\% $38 \mathrm{~g}$ or greater and central radiologic necrosis had a significantly inferior $2-y$ NRFS ( $53 \%$ vs. $77 \%$ and $45 \%$ vs. $95 \%$, respectively). Conclusion: The outcome of MNNs in patients with head and neck cancer receiving chemoradiotherapy/radiotherapy can be predicted according to radiologic necrosis and N-TLG40\% value. The 2 adverse features should be validated in future trials. In this way, patients can be treated alternatively or aggressively.
\end{abstract}

Key Words: ${ }^{18} \mathrm{~F}-\mathrm{FDG}$ PET/CT; CT; head and neck cancer; metastatic neck lymph node; radiotherapy

J Nucl Med 2015; 56:183-189

DOI: 10.2967/jnumed.114.148023

$\mathbf{O}$ rgan preservation with definitive chemoradiotherapy has become a treatment option in patients with head and neck cancers.

Received Aug. 31, 2014; revision accepted Dec. 26, 2014.

For correspondence or reprints contact: Chia-Hung Kao, Department of Nuclear Medicine and PET Center, China Medical University Hospital, No 2. Yur-Der Rd., Taichung 404, Taiwan.

E-mail: d10040@mail.cmuh.org.tw

${ }^{*}$ Contributed equally to this work.

Published online Jan. 22, 2015.

COPYRIGHT (c) 2015 by the Society of Nuclear Medicine and Molecular Imaging, Inc.
In the case of residual or recurrent diseases after initial therapy, salvage surgical intervention will be indicated. Although the status of neck disease is a major determinant of prognosis in head and neck cancers, the optimal management of the metastatic neck node (MNN) remains an issue of debate. A planned adjunctive neck dissection has been suggested in patients with N2 or N3 disease, which was based on some studies that demonstrated that radiotherapy combined with surgery might improve neck control rates, compared with 1 modality alone (1-3). However, several studies advocated surveillance of neck diseases because a complete remission can be achieved when assessing the response using ${ }^{18}$ F-FDG PET (4-7). In patients with regional recurrence after chemoradiotherapy/radiotherapy, salvage neck dissection would be possible but was associated with additional morbidity and worse prognosis $(8,9)$. Therefore, there is a need to identify pretreatment predictors that can foresee the outcome earlier when a decision of organ preservation or treatment modification should be discussed.

Although CT-based tumor volume or PET/CT has been used to predict treatment outcome in patients with head or neck cancers, there is still a lack of studies implementing comprehensive knowledge of the 2 images to identify imaging features that can be used to predict treatment outcomes for patients with MNN. Knowledge of imaging features that predict poor response to nonsurgical management could assist clinicians in selecting surgical therapy or considering dose-escalation schemes for patients with such high-risk features. To address this issue, we conducted a patient-based study to examine pretreatment parameters from both images.

\section{MATERIALS AND METHODS}

\section{Patient Population}

From January 2007 through June 2012, a cohort of 91 patients with pharyngeal cancers with histologic proof of squamous cell carcinoma, who had been treated with an organ preservation scheme at China Medical University Hospital, was included in this retrospective analysis after institutional review board (IRB) approval. The IRB (or equivalent) approved this retrospective study, and the requirement to obtain informed consent was waived (certificate number of local IRB, DMR99-IRB-010-1). The origin of the tumors was the oropharynx in 49 patients and hypopharynx in 42 patients. The median age was $52 \mathrm{y}$. All patients received pretreatment CT and PET/CT for initial staging within 4 wk before treatment was initiated. Because this study 
also aimed to recognize the association between nodal control and survival, a patient-based rather than a node-based analysis was performed. The characteristics of the 91 patients are shown in Table 1.

\section{Definition of CT-Based Parameters}

Each patient underwent a pretreatment contrast-enhanced CT scan of the neck with 3-mm-thick contiguous sections. Neck nodes were considered pathologic when their smallest-axis diameter was greater than $1 \mathrm{~cm}$. The CT images from the PACS were then transferred to a commercial planning system (Eclipse, version 8.1; Varian Medical System Inc.). Radiation oncologists then delineated the pretreatment gross tumor volume of the primary tumors and the MNN (10).

Three parameters including gross tumor volume of MNN (N-GTV), maximal nodal diameter, and radiologic central necrosis were retrieved from pretreatment CT images. Nodes in groups close together were scored as multiple node conglomerates. If there were multiple nodal sites on the CT scan, we selected the largest N-GTV for this patient-based analysis. The radiologic central necrosis was confirmed by radiologists. Because of lacking consistent consensus about extranodal spread, this parameter was not analyzed in this study.

\section{PET/CT Image Acquisition}

No patient had abnormal serum glucose levels before the PET/CT images were captured. All patients were required to fast for at least $4 \mathrm{~h}$ before ${ }^{18} \mathrm{~F}$-FDG PET/CT imaging. The images were captured using a PET/CT scanner (PET/CT-16 slice, Discovery STE; GE Medical System) approximately $60 \mathrm{~min}$ after the administration of $370 \mathrm{MBq}$ of ${ }^{18} \mathrm{~F}$-FDG. After the axial imaging range was determined, a spiral non-contrast-enhanced low-radiation dose CT scan (0.8-s rotation time, $120 \mathrm{kVp}$, variable $\mathrm{mA}$ with AutomA technique, 3.75-mm slice thickness, and 1.75:1 pitch) was acquired for anatomic reference and attenuation correction. PET emission images were then acquired sequentially after the CT scan at 1.5 min per field of view in 3-dimensional acquisition mode with an 11-slice overlap at the borders of the field of

TABLE 1

Patient Characteristics $(n=91)$

\begin{tabular}{|c|c|}
\hline Characteristic & Value \\
\hline Age (y) & 37-78 (median, 52) \\
\hline Sex & Male, 90; female, 1 \\
\hline Smoking & Yes, 80; no, 11 \\
\hline Betel nut squid & Yes, 64; no, 27 \\
\hline Alcoholism & Yes, 59; no, 32 \\
\hline \multicolumn{2}{|l|}{ Primary lesion site } \\
\hline Oropharynx & 49 \\
\hline Hypopharynx & 42 \\
\hline American Joint Committee on Cancer stage (24) & III, 10; IV, 81 \\
\hline T stage & T1, 7; T2, 36; T3, 27; T4, 21 \\
\hline $\mathrm{N}$ stage & $\mathrm{N} 1,15 ; \mathrm{N} 2,70 ; \mathrm{N} 3,6$ \\
\hline Total radiation dose (Gy) & 66-74 (median, 70) \\
\hline Overall radiation interval $(d)$ & 43-82 (median, 53) \\
\hline \multicolumn{2}{|l|}{ Concurrent chemotherapy or drug } \\
\hline Cisplatin-based & 70 \\
\hline Weekly cetuximab & 14 \\
\hline None & 7 \\
\hline \multicolumn{2}{|l|}{ PET/CT-related parameters } \\
\hline $\mathrm{N}-S U V_{\max }$ & $6.5 \pm 4.4$ (1.2-28.5); median, 6.1 \\
\hline N-MTV2.5 (mL) & $11.5 \pm 25.6$ (0.1-178); median, 3.0 \\
\hline N-MTV40\% (mL) & $10.0 \pm 18.4(1.0-153) ;$ median, 5.1 \\
\hline N-MTV50\% (mL) & $7.1 \pm 14.2$ (0.5-119); median, 3.6 \\
\hline N-TLG40\% (g) & $112.8 \pm 168.5(0-855.3) ;$ median, 38.0 \\
\hline N-TLG50\% (g) & $87.3 \pm 134.9(0-664.7) ;$ median, 29.0 \\
\hline \multicolumn{2}{|l|}{ CT-based volume parameters } \\
\hline N-GTV (mL) & $18.1 \pm 27.8(1-185) ;$ median, 8.9 \\
\hline Maximal diameter (cm) & $2.9 \pm 1.9$ (1.1-10.8); median, 2.4 \\
\hline Central necrosis & $51 / 91$ \\
\hline Follow up (mo) & 3-69 (median, 18) \\
\hline
\end{tabular}

$\mathrm{N}-\mathrm{MTV} 40 \%$ = pretreatment nodal metabolic tumor volume defined by $40 \%$ of maximal SUV; N-MTV50\% $=$ pretreatment nodal metabolic tumor volume defined by $50 \%$ of SUV $_{\max } ; \mathrm{N}-\mathrm{TLG} 40 \%=$ pretreatment nodal TLG defined by $40 \%$ of $\mathrm{SUV}$ max $; \mathrm{N}-\mathrm{TLG} 50 \%=$ pretreatment nodal TLG defined by $50 \%$ of $\mathrm{SUV}_{\max }$. 
view. The $\mathrm{CT}$ images were reconstructed onto a $512 \times 512$ matrix with a section thickness of $3.75 \mathrm{~mm}$ then reconstructed onto a $128 \times 128$ matrix and converted into 511-keV equivalent attenuation factors for attenuation correction of the corresponding PET emission images. The PET images were reconstructed using 3-dimensional iterative algorithms (VUE Point). The PET/CT workstation provided a quantification of ${ }^{18} \mathrm{~F}$-FDG uptake for standardized uptake value (SUV). This procedure has been described in our prior study (11). The maximum SUV of the target node was abbreviated as $\mathrm{N}-\mathrm{SUV}_{\max }$.

\section{Measurement of Metastatic Tumor Volume (MTV) and Total Lesion Glycolysis (TLG)}

We used the autosegmentation process of PET to define the volume of interest to reduce interobserver variability in image evaluation. MTVs and TLGs were measured from attenuation-corrected ${ }^{18} \mathrm{~F}-\mathrm{FDG}$ PET images using an SUV-based automated contouring program (Advantage Workstation Volume Share, version 2; GE Healthcare). The MTV was defined as the sum of the metabolic volumes of the primary tumors. The volume boundaries were sufficiently wide to incorporate each target lesion in the axial, coronal, and sagittal ${ }^{18} \mathrm{~F}$-FDG PET images. To define the contouring margins around the tumor, we used $\mathrm{SUV}_{\max }$ of 2.5 (MTV2.5) and 50\% of $\mathrm{SUV}_{\text {max }}$ (MTV50\%), as reported in our previous study (11). The TLG was calculated according to the following formula: $\mathrm{TLG}=\mathrm{SUV}_{\text {mean }} \times$ MTV (12). We used threshold levels that were equivalent for the MTVsthat is, TLG40\% and TLG50\%. The TLG40\% was labeled as pretreatment TLG defined by $40 \%$ of the maximal uptake of nodal TLG and the TLG50\% by $50 \%$ of the maximal uptake. Each patient had 2 sets of TLG: T-TLG for the primary tumor and N-TLG for the MNN. Similarly, the largest one was selected for the analysis in the case of multiple MNNs.

\section{Treatment}

Radiotherapy was performed using a sequential intensity-modulated radiotherapy technique (10). All patients received doses of 1.8 Gy daily, up to a total dose of between 68.4 and 73.8 Gy (median, 70.2 Gy). Two clinical target volumes (CTVs) were considered for various risks: CTV1 encompassed the primary tumor, MNNs, and the regions adjacent to the gross tumor, and CTV2 consisted of the ipsilateral or contralateral N0 regions at risk of harboring microscopic tumors. The dose delivered to CTV1/CTV2 during the first course was 50.4-54 Gy, with a further boost of 16.2-21.6 Gy to the CTV1 during the second course. Thus, the median cumulative doses of CTV1 and CTV2 were 70.2 and $54.0 \mathrm{~Gy}$, respectively. The median radiotherapy duration was $53 \mathrm{~d}$. Seventy patients received concurrent chemotherapy; their regimen consisted of cisplatin $\left(80-100 \mathrm{mg} / \mathrm{m}^{2}\right.$ on days 1,22 , and 43). Fourteen patients received combined cetuximab $\left(400 \mathrm{mg} / \mathrm{m}^{2}\right.$ loading dose and $250 \mathrm{mg} / \mathrm{m}^{2}$ ) weekly. Seven received radiotherapy alone.

\section{Follow-up}

According to the guidelines of the Response Evaluation Criteria in Solid Tumors (13), the initial treatment response was assessed by the CT scan done 1-2 mo after the completion of therapy. Patients were followed every 2-3 mo thereafter. A physical examination and laryngoscopy were performed during each follow-up examination, and a CT scan was obtained every 3-6 mo over 2 y. The definition of neck failure was based on the PET/CT, or progression of tumor on the CT scan. If patients had persistent tumors or recurrence after initial complete remission, salvage surgery was suggested if technically feasible and allowable by the condition of the patient.

\section{Statistical Analysis}

This study used the median values of the $\mathrm{N}_{-} \mathrm{SUV}_{\max }, \mathrm{N}-\mathrm{GTV}$, N-MTVs, and N-TLGs as cutoff points. The results of the statistical analysis are presented as the mean \pm SD. To examine the correlations between the parameters and recurrence, receiver-operating-characteristic curves were created to evaluate the optimal predictive performance among the MTVs and TLGs. The primary endpoints were the predictors for initial treatment response and nodal relapse-free survival (NRFS). The secondary endpoints were overall survival (OS) and disease-free survival (DFS). These rates were calculated using the Kaplan-Meier method. Logistic regression analysis was used to identify predictors for initial responders. Cox regression was performed to examine the effects of explanatory variables on OS, DFS, and NRFS. Although this study was to examine the impact of images on treatment outcome for MNN, PET/CT parameters describing primary and nodal tumors, as well as clinical parameters, were all included in the analysis when survivals were analyzed. Two-tailed tests were used, and $P$ values of less than 0.05 were considered statistically significant. All calculations were performed using SPSS 13.0 for Windows (SPSS Inc.).

\section{RESULTS}

\section{Parameter Measurement}

Four methods of calculating nodal MTV (N-MTV) and nodal TLG (N-TLG) values were retrieved for all patients. The mean $\mathrm{N}-\mathrm{GTV}$ was $18.1 \pm 27.8 \mathrm{~mL}$, and the mean $\mathrm{N}-\mathrm{SUV}_{\max }$ was $6.5 \pm 4.4$. The distributions of $\mathrm{N}-S U V_{\max }, \mathrm{N}-\mathrm{GTV}$, and various N-MTV and $\mathrm{N}-\mathrm{TLG}$ with respect to $\mathrm{N}$ classification are shown in Table 2. A trend of increasing values of PET/CT- or CT-related parameters was observed at an advanced $\mathrm{N}$ stage.

\section{Treatment Outcome}

According to the first CT scan after the treatment, 56 of the 91 MNNs (62\%) obtained a complete response, whereas $35(38 \%)$ had a partial response. After a median follow-up duration of $18 \mathrm{mo}$ (range, 6-69 mo), 37 patients were alive without known recurrent disease, and 16 patients had locoregional recurrence; however, they were alive after salvage or palliative treatment. Thirty-one patients died of tumor recurrence. Seven died of intercurrent diseases or other malignancies. Table 3 shows the detailed failure patterns of the cohort. In summary, 64 patients remained nodal relapse-free, whereas 27 patients experienced neck recurrence. Overall, the 2-y OS, DFS, and NRFS were 51\% (95\% confidence interval $[\mathrm{CI}], 42 \%-60 \%), 42 \%$ (95\% CI, 33\%-51\%), and $66 \%$ (95\% CI, 57\%-75\%), respectively.

\section{Comparison of Predictive Ability for Nodal Failure Among Different Threshold Methods}

The receiver-operating-characteristic curves were analyzed to compare the efficacy of various PET/CT-related parameters and threshold methods for determining the optimal approach for autosegmentation contouring. The results showed that N-MTV2.5 (pretreatment nodal metabolic tumor volume defined by SUV $=2.5$ ) and N-TLG40\% (40\% of the maximal uptake of nodal TLG) predicted the residual or recurrent nodes most accurately among the corresponding threshold methods (Supplemental Appendix 1; available at http://jnm.snmjournals.org). On the basis of the results, biologic tumor volumes using N-MTV2.5 and N-TLG40\% methods combined with $\mathrm{N}-\mathrm{SUV}_{\max }$ and CT-related parameters were selected for the analysis.

\section{Factors Associated with Initial Nodal Response}

According to the first follow-up CT scan, patients were classified into complete and partial responders. The logistic analysis showed N-GTV $\geq 8.9 \mathrm{~mL}$ ( $P=0.025$; odds ratio, 3.32; 95\% CI, 1.16-9.48) and radiologic central necrosis $(P<0.001$; odds ratio, $10.10 ; 95 \%$ CI, 3.03-34.48) were 2 factors associated with partial remission of the MNNs (Supplemental Appendix 2). 
TABLE 2

Distribution of CT- and PET/CT-Related Parameters with Respect to N Classification

\begin{tabular}{|c|c|c|c|c|c|}
\hline Variable & N1 & N2 & N3 & All & Median \\
\hline \multicolumn{6}{|l|}{$\begin{array}{l}\text { CT-based } \\
\text { parameters }\end{array}$} \\
\hline $\mathrm{N}-\mathrm{GTV}(\mathrm{mL})$ & $4.1 \pm 2.8(1.7-11.2)$ & $14 \pm 14.1(1.0-64.5)$ & $101 \pm 46.3(51.2-185.4)$ & $18.1 \pm 27.8(1-185)$ & 8.9 \\
\hline $\begin{array}{l}\text { Maximal } \\
\text { diameter }(\mathrm{cm})\end{array}$ & $1.7 \pm 0.5(1.2-2.6)$ & $2.7 \pm 1.5(1.1-7.1)$ & $7.6 \pm 1.9(5.8-10.8)$ & $2.9 \pm 1.9(1.1-10.8)$ & 2.4 \\
\hline Central necrosis & $4 / 15$ & $41 / 70$ & $6 / 6$ & $51 / 91$ & \\
\hline \multicolumn{6}{|l|}{$\begin{array}{l}\text { PET/CT-based } \\
\text { parameters }\end{array}$} \\
\hline $\mathrm{N}-S U V_{\max }$ & $4.2 \pm 2.3(1.2-8.6)$ & $6.9 \pm 4.4(1.3-28.5)$ & $8.6 \pm 5.8(1.3-15.9)$ & $6.5 \pm 4.4(1.2-28.5)$ & 6.1 \\
\hline N-MTV2.5 (mL) & $2.4 \pm 2.7(0.1-8.9)$ & $8.3 \pm 11.8(0.1-59.1)$ & $72.3 \pm 71.1(0.1-178)$ & $11.5 \pm 25.6(0.1-178)$ & 3.0 \\
\hline N-MTV40\% (mL) & $6.2 \pm 2.9(2.1-12.8)$ & $7.3 \pm 6.9(1.0-32.5)$ & $51 \pm 56.9(4.0-153)$ & $10.0 \pm 8.4(1.0-153)$ & 5.1 \\
\hline N-MTV50\% (mL) & $3.8 \pm 1.4(1.5-6.8)$ & $5.1 \pm 5.2(0.5-25.5)$ & $8.4 \pm 44.4(3.6-119)$ & $7.1 \pm 14.2(0.5-119)$ & 3.6 \\
\hline N-TLG40\% (g) & $60.4 \pm 74.9(0-226.8)$ & $100.4 \pm 150.6(0-855.3)$ & $388.3 \pm 283.9(0-763.2)$ & $12.8 \pm 168.5(0-855.3)$ & 37.9 \\
\hline N-TLG50\% (g) & $45.2 \pm 56.2(0-170.7)$ & $77.7 \pm 119(0-664.7)$ & $305.3 \pm 244.8(0-634.7)$ & $87.3 \pm 134.9(0-664.7)$ & 29 \\
\hline
\end{tabular}

$\mathrm{N}-\mathrm{MTV} 40 \%$ = pretreatment nodal metabolic tumor volume defined by $40 \%$ of maximal SUV; N-MTV50\% $=$ pretreatment nodal metabolic tumor volume defined by $50 \%$ of SUV $\max ; \mathrm{N}-\mathrm{TLG} 40 \%=$ pretreatment nodal TLG defined by $40 \%$ of $\mathrm{SUV}$ max $; \mathrm{N}-\mathrm{TLG} 50 \%=$ pretreatment nodal TLG defined by $50 \%$ of $S U V_{\max }$.

All values are mean $\pm S D$, with range in parentheses.

\section{Prognostic Factors for Nodal Relapse and Survival}

The Cox regression analysis showed that $\mathrm{N}-\mathrm{TLG} 40 \% \geq 38 \mathrm{~g}(P=$ 0.03 ; hazard ratio [HR], 2.63; 95\% CI, 1.10-6.30) and radiologic central necrosis $(P=0.001 ; \mathrm{HR}, 10.99 ; 95 \% \mathrm{CI}, 2.56-47.62)$ were 2 predictors for neck recurrence (Table 4 ). The 2-y NRFS for patients who had tumors with N-TLG40\% $\geq 38 \mathrm{~g}$ and $<38 \mathrm{~g}$ was $53 \%$ and $77 \%$, respectively (Fig. 1). Patients with radiologic central necrosis had a lower 2-y NRFS than those without this feature (45\% vs. $95 \%$; Fig. 2). Although a large N-GTV, or a higher $\mathrm{N}_{-} \mathrm{SUV}_{\max }$, was also associated with higher risk of recurrence, there was no statistical significance in the multivariate analyses. In addition, no difference of NRFS curves was found between the origin of the primary tumors (Supplemental Appendix 3). When N-TLG40\% $\geq 38 \mathrm{~g}$ was used as a cutoff to predict nodal failure, the sensitivity, specificity, and accuracy were $44 \%, 85 \%$, and $65 \%$, respectively. When the central necrosis was applied to forecast recurrence, the sensitivity, specificity, and accuracy were $47 \%, 93 \%$, and $67 \%$.

As also shown in Table 4, 2 predictors for inferior OS were T3T4 $(P=0.01 ; \mathrm{HR}, 2.68 ; 95 \% \mathrm{CI}, 1.27-5.64)$ and central necrosis $(P=0.02$; HR, 2.59; 95\% CI, 1.13-5.26). T-MTV2.5 (metabolic tumor volume of primary tumor defined by SUV $=2.5$ ) showed a marginal impact on OS. The prognosticators of DFS were T3-T4 $(P=0.001 ; \mathrm{HR}, 3.63 ; 95 \% \mathrm{CI}, 1.75-7.50)$, central necrosis $(P<$ 0.001 ; HR, 3.62; 95\% CI, 1.79-7.35), and N-TLG40\% $\geq 38 \mathrm{~g}$ $(P=0.02$; HR, 2.12; 95\% CI, 1.13-4.00).

\section{Subgroup Analysis in Patients with N2-N3 Neck Disease}

Because some studies advocated a planned neck dissection for patients with N2-N3 disease, we performed a subgroup analysis for these patients $(n=76)$ to examine the performance of the parameters mentioned above. The Cox regression analysis showed a similar finding that N-TLG40\% $\geq 38 \mathrm{~g}(P=0.04$; HR, 2.22; 95\% CI, 1.04-4.79) and central necrosis $(P=0.002$; HR, 4.99; 95\% CI, 1.83-13.69) were 2 prognostic factors for nodal relapse.

\section{Risk Stratification of Nodal Relapse According to Major Adverse Factors}

When nodal diseases were stratified with the 2 prognostic factors, patients could be evenly stratified with 3 groups. Group A

TABLE 3

Patient Outcome $(n=91)$

\begin{tabular}{|c|c|c|}
\hline Outcome & $\begin{array}{l}\text { Total no. } \\
\text { of patients }\end{array}$ & $\begin{array}{l}\text { Number } \\
\text { of patients } \\
\text { in each } \\
\text { subgroup }\end{array}$ \\
\hline Alive without evidence of recurrence & 37 & \\
\hline $\begin{array}{l}\text { Alive with evidence of disease } \\
\text { recurrence }\end{array}$ & 16 & \\
\hline Primary relapse alone & & 5 \\
\hline Neck lymph node relapse alone & & 4 \\
\hline Primary and lymph node relapse & & 5 \\
\hline Distant metastasis alone & & 2 \\
\hline Died of cancer & 31 & \\
\hline $\begin{array}{l}\text { Primary and neck lymph node } \\
\text { relapse }\end{array}$ & & 12 \\
\hline Primary relapse alone & & 9 \\
\hline $\begin{array}{l}\text { Primary relapse and distant } \\
\text { metastasis }\end{array}$ & & 1 \\
\hline Neck lymph node relapse alone & & 2 \\
\hline Distant metastasis alone & & 3 \\
\hline $\begin{array}{l}\text { Primary, neck, and distant } \\
\text { metastasis }\end{array}$ & & 4 \\
\hline $\begin{array}{l}\text { Died of intercurrent diseases or } \\
\text { other malignancies }\end{array}$ & 7 & \\
\hline
\end{tabular}


TABLE 4

Association Between PET/CT- and CT-Based Tumor Parameters and Survivals Using Cox Regression Model

\begin{tabular}{|c|c|c|c|c|c|c|c|c|c|}
\hline \multirow[b]{2}{*}{ Variable } & \multicolumn{3}{|c|}{ OS } & \multicolumn{3}{|c|}{ DFS } & \multicolumn{3}{|c|}{ NRFS } \\
\hline & $\mathrm{HR}$ & $95 \% \mathrm{Cl}$ & $P$ & $\mathrm{HR}$ & $95 \% \mathrm{Cl}$ & $P$ & $\mathrm{HR}$ & $95 \% \mathrm{Cl}$ & $P$ \\
\hline \multicolumn{10}{|l|}{ TNM classification } \\
\hline \multicolumn{10}{|l|}{ T stage } \\
\hline T1-2 vs. T3-4 & 2.68 & $1.27-5.64$ & 0.01 & 3.63 & $1.75-7.50$ & 0.001 & 2.40 & $0.90-6.46$ & 0.07 \\
\hline \multicolumn{10}{|l|}{$\mathrm{N}$ stage } \\
\hline N1-2 vs. N3 & 1.40 & $0.36-4.50$ & 0.71 & 1.34 & $0.4-4.03$ & 0.60 & 1.25 & $0.21-3.01$ & 0.56 \\
\hline N1 vs. N2-3 & 1.02 & $0.40-2.62$ & 0.98 & 1.09 & $0.44-2.74$ & 0.85 & 1.43 & $0.38-5.40$ & 0.60 \\
\hline \multicolumn{10}{|l|}{ CT-related parameters for MNN } \\
\hline Central necrosis, no vs. yes & 2.59 & $1.13-5.26$ & 0.02 & 3.62 & $1.79-7.35$ & $<0.001$ & 10.99 & $2.56-47.62$ & 0.001 \\
\hline Maximal diameter $(\mathrm{cm}),<2.4$ vs. $\geq 2.4$ & 1.24 & $0.48-3.27$ & 0.66 & 2.16 & $0.78-6.01$ & 0.14 & 1.80 & $0.55-5.92$ & 0.34 \\
\hline N-GTV (mL), <8.9 vs. $\geq 8.9$ & 1.56 & $0.21-1.91$ & 0.42 & 1.01 & $0.41-2.47$ & 0.98 & 1.02 & $0.25-4.22$ & 0.98 \\
\hline \multicolumn{10}{|l|}{ PET/CT-related parameters } \\
\hline $\mathrm{N}-\mathrm{TLG} 40 \%(\mathrm{~g}),<38.0$ vs. $\geq 38.0 \mathrm{~g}$ & 1.71 & $0.77-3.78$ & 0.19 & 2.12 & $1.13-4.00$ & 0.02 & 2.63 & $1.10-6.30$ & 0.03 \\
\hline N-SUV $\max ,<6.1$ vs. $\geq 6.1$ & 1.68 & $0.72-3.92$ & 0.23 & 1.36 & $0.55-3.36$ & 0.51 & 1.57 & $0.22-1.80$ & 0.40 \\
\hline N-MTV2.5 (mL), <3.0 vs. $\geq 3.0$ & 1.62 & $0.75-3.50$ & 0.22 & 1.20 & $0.57-2.56$ & 0.64 & 1.19 & $0.51-2.75$ & 0.69 \\
\hline T-TLG40\% (g), $<53.3$ vs. $\geq 53.3$ & 1.09 & $0.47-2.09$ & 0.97 & 1.75 & $0.71-4.32$ & 0.22 & 1.72 & $0.70-4.24$ & 0.24 \\
\hline T-SUV $_{\max },<10.7$ vs. $\geq 10.7$ & 1.39 & $0.61-3.22$ & 0.43 & 1.55 & $0.58-4.09$ & 0.38 & 1.67 & $0.64-4.33$ & 0.29 \\
\hline T-MTV2.5 (mL), $<14.5$ vs. $\geq 14.5$ & 2.43 & $0.99-6.05$ & 0.06 & 1.11 & $0.38-2.72$ & 0.88 & 0.93 & $0.36-2.42$ & 0.88 \\
\hline $\begin{array}{l}\text { Primary tumor origin, oropharynx vs. } \\
\text { hypopharynx }\end{array}$ & 1.11 & $0.56-2.17$ & 0.77 & 0.99 & $0.47-2.13$ & 0.99 & 0.98 & $0.46-2.09$ & 0.95 \\
\hline
\end{tabular}

T-TLG40\% = pretreatment primary TLG defined by $40 \%$ of SUV $_{\max } ; \mathrm{T}_{-} \mathrm{SUV}_{\max }=$ pretreatment maximum SUV of primary tumor; T-MTV2.5 = pretreatment primary metabolic tumor volume defined by SUV $=2.5$.

This study used median values of T-SUV $\max$, T-MTVs, and T-TLGs as cutoff points.

comprised 28 patients without any adverse features. Group C included 33 patients having both risk factors, and group B consisted of 30 patients having 1 of the 2 . The estimated 2-y NRFS was $92 \%$ for group A, $78 \%$ for group B, and 33\% for group C (Fig. 3).

\section{DISCUSSION}

An advanced nodal stage in patients with head and neck cancers is a well-known adverse factor for survival (14). When investigating the prognostic role of the image-related factors, it would be appropriate to examine all the parameters derived from the primary tumors and MNNs. Because of the insufficiency of using $\mathrm{T}$ or $\mathrm{N}$ classification alone in assessing the final outcome, we previously reported the clinical implication of CT- and PET/CT-based findings on the control of primary tumor (11). This study further disclosed the efficacy of implementing image-related factors on neck control. Before the evolution of organ preservation, neck nodes could be usually dissected at the same time as excision of primary tumors. To date, neck dissection is commonly reserved for those with residual or recurrent disease after the initial therapy. Therefore, the evaluation of nodal response became crucial to the adequate performance of salvage neck dissection. Although posttreatment CT or PET had a high accuracy in determining the regional control $(4-7,15)$, early recognition of patients at risk for nodal failure after curative nonsurgical treatment can optimize the individual treatment schemes by reducing the number of patients undergoing unsuitable treatment.
The role of several prognostic factors for nodal recurrence after radiotherapy has been investigated. Previous studies showed that nodal size, radiologic signs of extranodal spread, and central necrosis are prognostic factors for regional control (8,9,16-18). Through comprehensive CT- and PET/CT-related

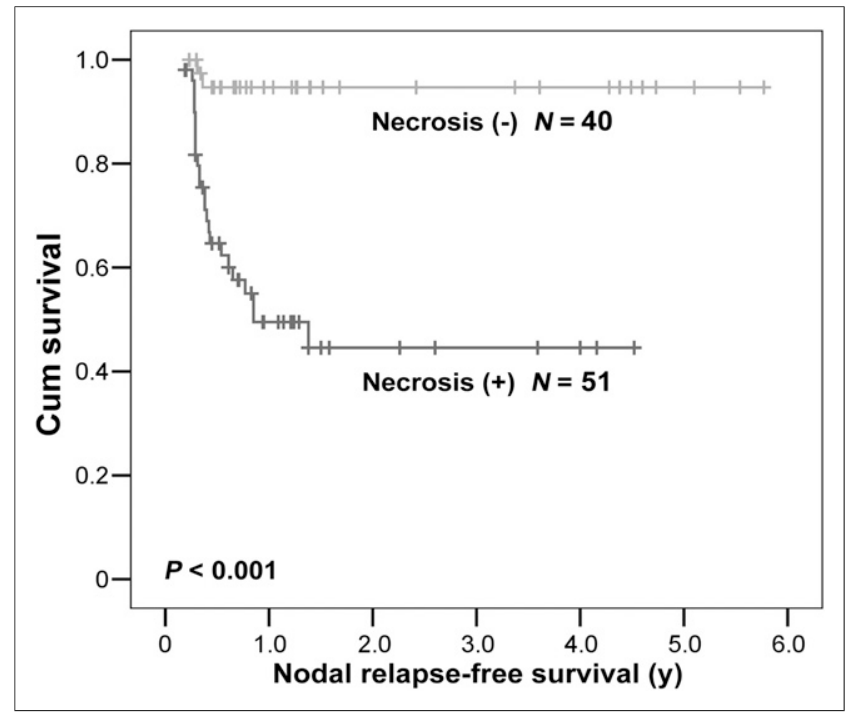

FIGURE 1. NRFS according to central necrosis on CT scan $(P<0.001)$. 


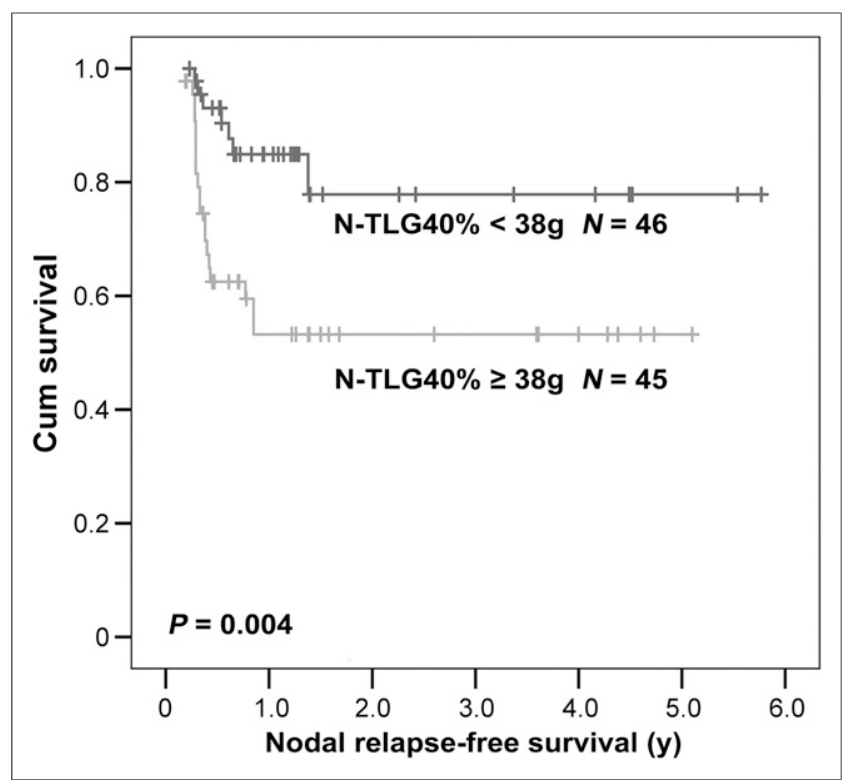

FIGURE 2. NRFS according to nodal TLG $40 \% \geqq 38 \mathrm{~g}$ and $<38 \mathrm{~g}(P=$ $0.004)$.

parameters with various threshold methods, we first showed that the risk of residual or recurrent neck diseases can be stratified by combining N-TLG40\% with radiologic central necrosis. Particularly for patients with any MNNs categorized as group C, alternative modalities can be considered before a decision of definitive chemoradiotherapy/radiotherapy. In addition, for patients having chosen an organ-preservation scheme, a planned neck dissection for the high-risk patients can be discussed earlier. Certainly, further studies are warranted to test our results because of the low sensitivity presented in this study.

A unique advantage of ${ }^{18} \mathrm{~F}$-FDG PET/CT is its ability to automatically create a tumor contour using quantitative information on glucose uptake within the tumor. In patients with head and neck cancers receiving definitive chemoradiotherapy/radiotherapy, the use of pretreatment biologic tumor volume as a predictive factor is not novel. However, few studies have compared comprehensive volumetric and threshold methods to define the optimal approach for MNN. Using receiver-operating characteristic analysis, we first examined the efficacy of various threshold methods for determining the best approach. Then, the predictive abilities of these methods were compared with those derived from CT-related and clinical parameters. To identify the optimal cutoff values for which the sum of sensitivity and specificity was the greatest, undoubtedly, it is essential to enroll more participants prospectively and to use standardized protocols for ${ }^{18} \mathrm{~F}-\mathrm{FDG}$ PET acquisition and processing. Nonetheless, this is a pilot study to clarify that the N-TLG40\% method was better than nodal volume or MTV approaches in predicting NRFS or DFS for these patients. Although other threshold approaches for N-TLG or N-MTV failed to show a superior predictive power in NRFS, compared with the N-TLG40\%, all tested threshold methods exhibited a biologic phenotype trend for nodal recurrence. In the era of considering dose escalation for ${ }^{18}$ F-FDG-avid tumors, Jeong et al. (19) reported a novel outcomeequivalent dose-analysis method to estimate the dose-response modifying effect of ${ }^{18} \mathrm{~F}-\mathrm{FDG}$ uptake variation. The results of their study provided a rational starting point for the selection of intensity-modulated radiotherapy to be used for ${ }^{18} \mathrm{~F}-\mathrm{FDG}$-avid tumors. Their study indicated ${ }^{18} \mathrm{~F}-\mathrm{FDG}$-avid tumors are likely to require $10 \%-30 \%$ more dose than ${ }^{18} \mathrm{~F}-\mathrm{FDG}$-nonavid tumors to reach equal response rates. Our study presented a clinical basis when considering dose-escalating scheme to the nodes.

A previous study showed that the presence of central hypodense zones on CT correlated well with a high incidence of nodal necrosis (20). On the basis of the same radiologic definition, we demonstrated that nodal control and survivals were significantly associated with central necrosis, as described in previous studies $(8,18)$. Interestingly, our data also showed that radiologic central necrosis was positively associated with several CT and PET/CT parameters including N-GTV, SUV ${ }_{\max }$, and N-TLG. Theoretically, the hypodense necrotic zones on CT contrast images imply areas of hypovascularity and might harbor hypoxic cells, which could lead to the negative impact on nodal control as hypoxic cells are less radiosensitive (18). Nakajima et al. (21) analyzed tumor cells grown as xenograft in nude mice after the identification of the metabolic response to hypoxia and found 2-deoxyglucose uptake in hypoxic regions of the tumors was approximately 2 times higher than in the whole tumor. They concluded that hypoxia is associated with increased intratumoral metabolic heterogeneities on ${ }^{18}$ F-FDG PET. Given that the metabolic heterogeneity within the tumors might be an indicator of tumor hypoxia, it is warranted to correlate the heterogeneities with clinical outcome in the future. In addition, the radiologic necrosis needs to be scored, and the association between the score and extent of heterogeneities of ${ }^{18}$ F-FDG uptake should be investigated further.

This study was subject to numerous limitations, such as a lack of MR image information. The ability of diffusion-weighted MR imaging in improving target volume delineation, early tumor response assessment, and differentiation between normal posttreatment changes suggests an important clinical role in radiotherapy (22). It would be interesting to compare the impact of diffusion-weighted MR imaging on the treatment outcome. In addition, posttreatment PET/CT was not routinely performed for relevant prognostic information. Thus, the changes of PET/CTrelated parameters before and after therapy could not be assessed accordingly. Finally, the impact of human papillomavirus (HPV)related oropharyngeal cancer on prognosis has gained great

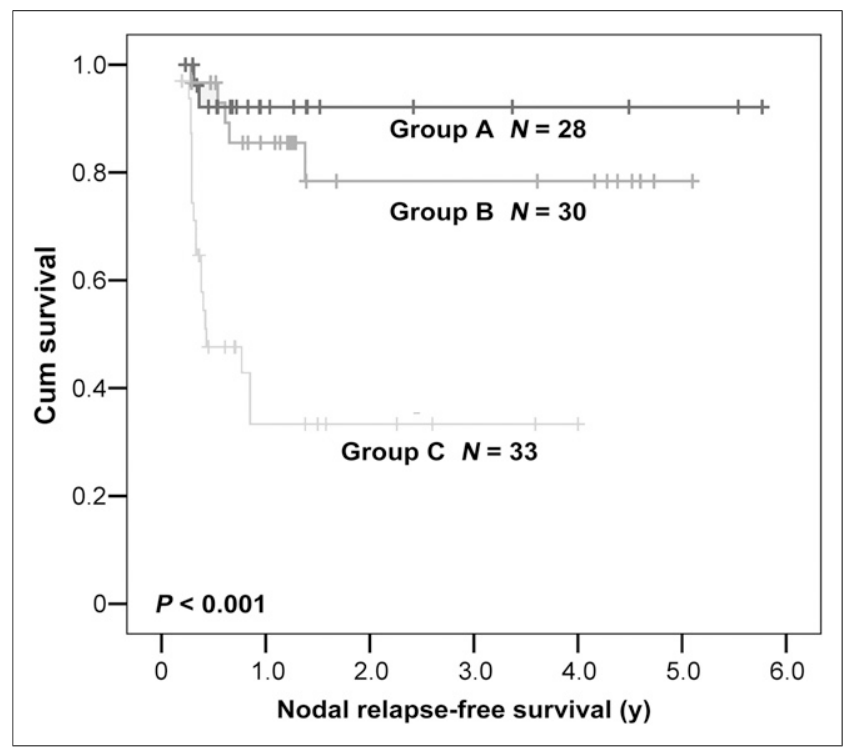

FIGURE 3. NRFS according to nodal groups $(P<0.001)$. 
interest. The effect on prognosis might be stronger than many other factors investigated before, including stage or ${ }^{18} \mathrm{~F}$-FDG uptake. Despite the lower prevalence of human HPV-related oropharyngeal cancer in Asia $(22,23)$, the results would be more robust if information about the HPV status could be included in this analysis. Future studies must use more imaging studies and information of HPV status and adjust for potential confounders in the analysis. In addition, ${ }^{18}$ F-FDG uptake variability in human tumors may be an indicator of tumor hypoxia, and prognosis, and therefore could be validated in prospective clinical trials. On the basis of our finding, we recommend that treatment modification or an alternative treatment can be considered for patients with a pretreatment $\mathrm{N}-\mathrm{TLG} 40 \% \geq 38 \mathrm{~g}$ or radiologic central necrosis. Such treatment modification may include dose escalation, novel cytotoxic drugs, or the use of adjunctive neck dissection. In this manner, patients for individual treatment schemes can be selected more appropriately.

\section{CONCLUSION}

This pilot study shows that the control rate of MNN in patients with head and neck cancer receiving radiotherapy/chemoradiotherapy for organ preservation can be predicted according to radiologic central necrosis on CT scan and N-TLG40\% value on $\mathrm{PET} / \mathrm{CT}$. The result should be validated in future clinical trials. In this way, patients with the adverse features can be considered for alternative or aggressive treatment.

\section{DISCLOSURE}

The costs of publication of this article were defrayed in part by the payment of page charges. Therefore, and solely to indicate this fact, this article is hereby marked "advertisement" in accordance with 18 USC section 1734. We thank the grant support (CMU98-C-13) of the China Medical University and Taiwan Ministry of Health and Welfare Clinical Trial and Research Center of Excellence (MOHW103TDU-B-212-113002), health and welfare surcharge of tobacco products, and China Medical University Hospital Cancer Research Center of Excellence (MOHW103-TD-B-111-03, Taiwan). No other potential conflict of interest relevant to this article was reported.

\section{REFERENCES}

1. Brizel DM, Prosnitz RG, Hunter S, et al. Necessity for adjuvant neck dissection in setting of concurrent chemoradiation for advanced head and-neck cancer. Int J Radiat Oncol Biol Phys. 2004;58:1418-1423.

2. Mendenhall WM, Amdur RJ, Stringer SP, Villaret DB, Cassisi NJ. Radiation therapy for squamous cell carcinoma of the tonsillar region: a preferred alternative to surgery? J Clin Oncol. 2000;18:2219-2225.

3. Lavertu P, Adelstein DJ, Saxton JP, et al. Management of the neck in a randomized trial comparing concurrent chemotherapy and radiotherapy with radiotherapy alone in resectable stage III and IV squamous cell head and neck cancer. Head Neck. 1997;19:559-566.

4. Ong SC, Schoder H, Lee NY, et al. Clinical utility of ${ }^{18} \mathrm{~F}-\mathrm{FDG}$ PET/CT in assessing the neck after concurrent chemoradiotherapy for locoregional advanced head and neck cancer. J Nucl Med. 2008;49:532-540.
5. Porceddu SV, Jarmolowski E, Hicks RJ, et al. Utility of positron emission tomography for the detection of disease in residual neck nodes after (chemo) radiotherapy in head and neck cancer. Head Neck. 2005;27:175-181.

6. Connell CA, Corry J, Milner AD, et al. Clinical impact of, and prognostic stratification by, F-18 FDG PET/CT in head and neck mucosal squamous cell carcinoma. Head Neck. 2007;29:986-995.

7. Goenka A, Morris LG, Rao SS, et al. Long-term regional control in the observed neck following definitive chemoradiation for node-positive oropharyngeal squamous cell cancer. Int J Cancer. 2013;133:1214-1221.

8. Vergeer MR, Doornaert P. C Leemans CR, Buter J, Slotman BJ, Langendijk JA. Control of nodal metastases in squamous cell head and neck cancer treated by radiation therapy or chemoradiation. Radiother Oncol. 2006;79:39-44.

9. Hermans R, Op de beeck K, Van den Bogaert W, et al. The relation of CT determined tumor parameters and local and regional outcome of tonsillar cancer after definitive radiation treatment. Int J Radiat Oncol Biol Phys. 2001;50:37-45.

10. Yang SN, Liao CY, Chen SW, et al. Clinical implications of the tumor volume reduction rate in head-and-neck cancer during definitive intensity-modulated radiotherapy for organ preservation. Int J Radiat Oncol Biol Phys. 2011;79: 1096-1103.

11. Kao CH, Lin SC, Hsieh TC, et al. Use of pretreatment metabolic tumor volumes to predict the outcome of pharyngeal cancer treated by definitive radiotherapy. Eur J Nucl Med Mol Imaging. 2012;39:1297-1305.

12. Larson SM, Erdi Y, Akhurst T, et al. Tumor treatment response based on visual and quantitative changes in global tumor glycolysis using PET-FDG imaging: the visual response score and the change in total lesion glycolysis. Clin Positron Imaging. 1999;2:159-171.

13. Eisenhauer EA, Therasse P, Bogaerts J, et al. New response evaluation criteria in solid tumours: revised RECIST guideline (version 1.1). Eur J Cancer. 2009;45:228-247.

14. Brockstein B, Haraf DJ, Rademaker AW, et al. Patterns of failure, prognostic factors and survival in locoregionally advanced head and neck cancer treated with concomitant chemoradiotherapy: a 9-year, 337-patient, multi-institutional experience. Ann Oncol. 2004;15:1179-1186.

15. Liauw SL, Mancuso AA, Amdur RJ, et al. Postradiotherapy neck dissection for node-positive head and neck cancer: the use of computed tomography to manage the neck. J Clin Oncol. 2006;24:1421-1427.

16. Prada Gomez PJ, Rodriguez R, Rijo GJ, et al. Control of neck nodes in squamous cell carcinoma of the head and neck by radiotherapy: prognostic factors. Clin Otolaryngol Allied Sci. 1992;17:163-169.

17. Bataini JP, Bernier J, Asselain B, et al. Primary radiotherapy of squamous cell carcinoma of the oropharynx and pharyngolarynx: tentative multivariate modelling system to predict the radiocurability of neck nodes. Int J Radiat Oncol Biol Phys. 1988;14:635-642.

18. Munck JN, Cvitkovic E, Piekarski JD, et al. Computed tomographic density of metastatic lymph nodes as a treatment-related prognostic factor in advanced head and neck cancer. J Natl Cancer Inst. 1991;83:569-575.

19. Jeong J, Setton JS, Lee NY, Oh JH, Deasy JO. Estimate of the impact of FDGavidity on the dose required for head and neck radiotherapy local control. Radiother Oncol. 2014;111:340-347.

20. Sham JS, Cheung YK, Choy D, Chan FL, Leong L. Computed tomography evaluation of neck node metastases from nasopharyngeal carcinoma. Int J Radiat Oncol Biol Phys. 1993;26:787-792.

21. Nakajima EC, Laymon C, Oborski M, et al. Quantifying metabolic heterogeneity in head and neck tumors in real time: 2-DG uptake is highest in hypoxic tumor regions. PLoS One 2014; 15;9:e102 452.

22. Tsien C, Cao Y, Chenevert T. Clinical applications for diffusion magnetic resonance imaging in radiotherapy. Semin Radiat Oncol. 2014;24:218-226.

23. Chien CY, Su CY, Fang FM, et al. Lower prevalence but favorable survival for human papillomavirus-related squamous cell carcinoma of tonsil in Taiwan. Oral Oncol. 2008;44:174-179.

24. Edge S, Byrd DR, Compton CC, Fritz AG, Greene FL, Trotti A (Eds.). AJCC Cancer Staging Manual. 7th ed. New York, NY: Springer; 2010. 